\title{
FIP/WHO/CHINA Bioequivalence and Hands-on-Dissolution Workshop Report Beijing, China, April 12-13, 2004 Shanghai, China, April 15-16, 2004
}

\author{
Vinod P. Shah, Ph. D. \\ FIP Scientific Secretary
}

$\mathrm{T}$ he FIP/WHO workshops on "Bioequivalence and Hands-on-Dissolution" in Beijing and Shanghai, China were organized by FIP-BPS/SIG BA-BE Dissolution Working Group and the National Institute for the control of Pharmaceuticals \& Biological Products (NICPBP), China. The co-chairs of the workshop were Dr. Vinod P. Shah, Scientific Secretary FIP and Professor Shaohong Jin, the executive head of NICPBP. The planning committee of the workshop included Dr. Martin Siewert (Aventis, Germany), Dr. Hans Junginger (Immediate past Scientific Secretary, FIP), Dr. Sabine Kopp (WHO), Dr. Zhao Lili (State and Food and Drug Administration, (SFDA) China), and Jiye Huang (CCPIE, China). The scientific part of the workshop was organized by Prof. Jin, with the other logistics handled by Ms. Jing Sun of CCPIE.

The international faculty of the workshop included Vinod Shah (USA), Vivian Gray (USA), Cindy Brown (USA), Sabine Kopp (Geneva), Kamal Midha (Canada), Hans Junginger (Germany) and Roy Hanson (USA). The faculty was requested to provide their PowerPoint presentations in advance to be translated into Chinese. As a result of this effort the handouts contained both the English and the translated Chinese versions. Each speaker was assigned a translator who worked with the speaker to review and understand the presentation.

The workshop program began with a Greetings and Welcome from Jiye Huang from CCPIE, Shahong Jin from NICPBP, Vinod Shah from FIP, Sabine Kopp from WHO and Zhao Lilli from SFDA. Lixia Ding from NICPBP presided as the moderator.

The scientific program began with a presentation by Professor Jin. He presented the challenges in dissolution testing, bioequivalence and registration in China. He also discussed information about dissolution test method sampling that will be included in ChP2005 and the proposal of a self-reference method for dissolution test. The next presentation was by Vinod Shah who discussed the design of a dissolution test, FDA dissolution guidances, the importance of dissolution test and its impact. Vivian Gray then discussed the design and calibration of dissolution test equipment, appropriate method and procedure for calibrating the equipment and use of the calibrator as a system suitability test. Cindy Brown discussed validation of dissolution test parameters and the importance of selecting and optimizing appropriate dissolution methodology.

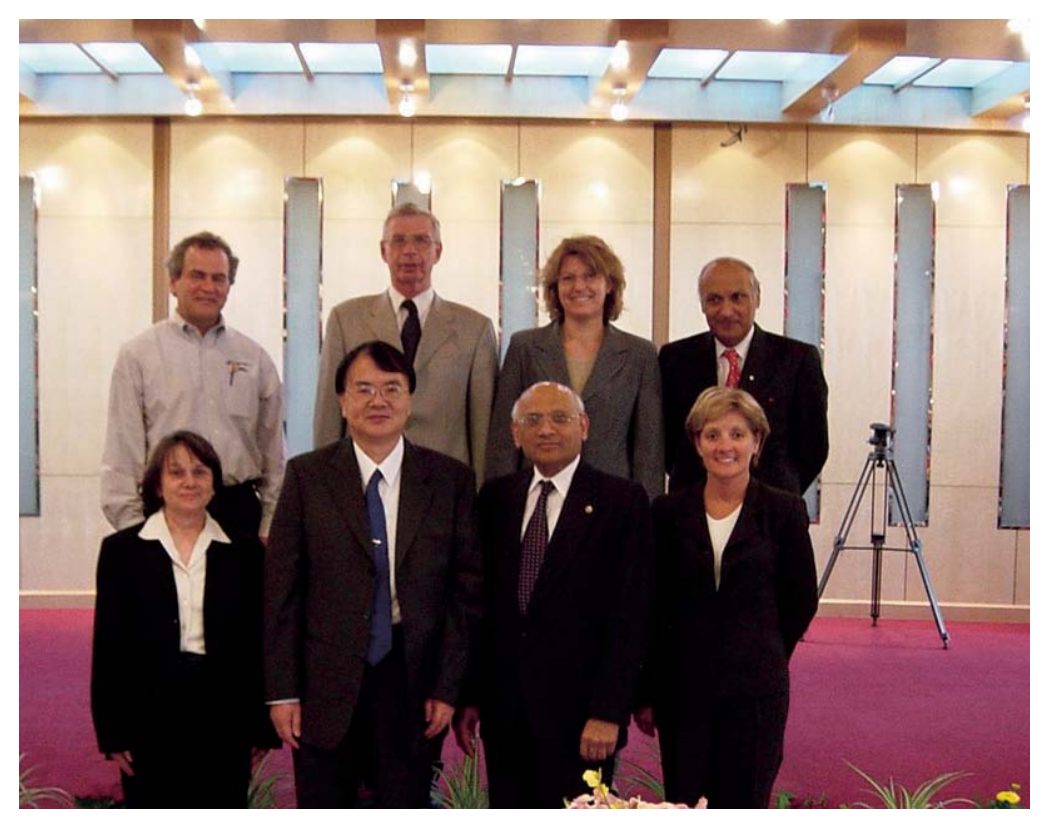

Faculty_left to right, starting at the top: Roy Hanson, Hans Junginger, Sabine Kopp, Kamal Midha, Vivian Gray, Professor Jin, Vinod Shah, Cindy Brown 
Sabine Kopp discussed the WHO general chapter on dissolution testing for inclusion in the International Pharmacopoeia and WHO procedures for drug quality control specifications. She discussed the proposal of a Biopharmaceutics Classification System (BCS) conforming dissolution test methods for BCS class 1 drug products. The dissolution test method proposed will require the drug product to dissolve $85 \%$ in 30 minutes using the paddle method at $75 \mathrm{rpm}$ in $500 \mathrm{ml}$ of $\mathrm{pH} 6.8$ buffer medium.

For ease of operation, the participants were divided into two groups in the afternoon. Roy Hanson, Cindy Brown and Vivian Gray demonstrated the setting and testing of the dissolution equipment provided by the

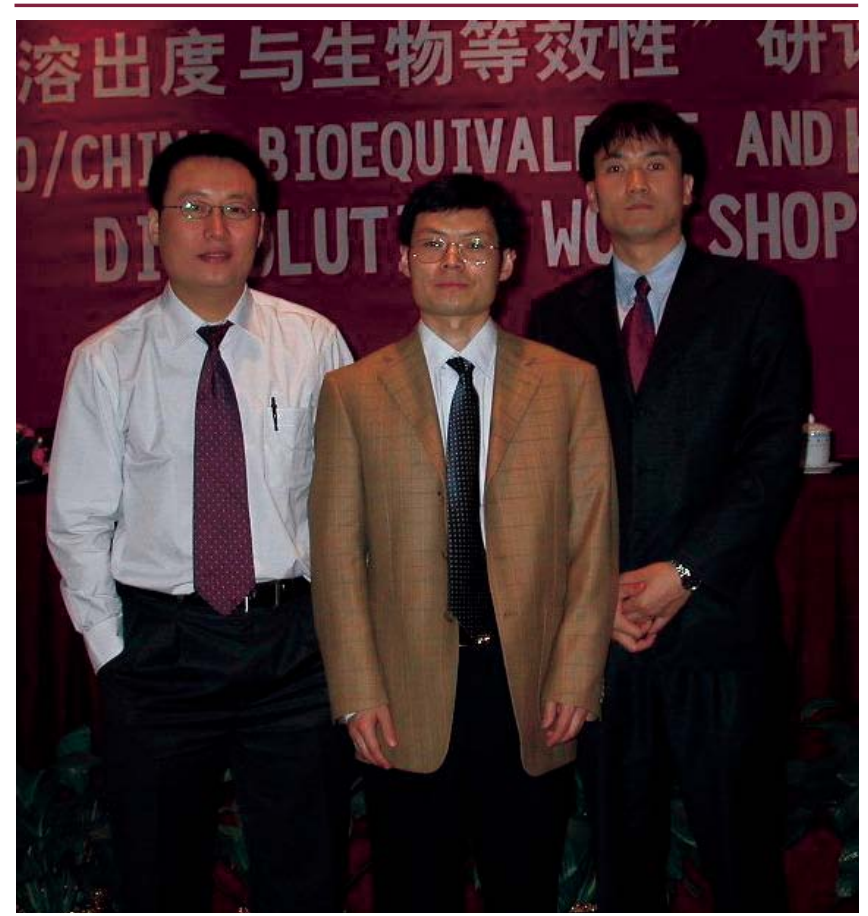

Interpreters -From left to right: Wang Jia-wei, Ning Bao-ming, and Xu Ming-zhe.
Hanson Research Company. The other group of participants enjoyed the $Q$ and $A$ session.

On the next day, Vinod Shah discussed BCS and Biowaiver guidance. The requirements for biowaiver for IR dosage forms in general and specifically BCS Class 1 and ER dosage forms, dissolution as a Quality Control (QC) test $v s$ a Bioequivalence (BE) test and dissolution profile comparison were discussed. Hans Junginger discussed the development of novel dosage forms and identified ideal attributes of a good drug delivery system. Different types of Controlled Release (CR) dosage forms, drug release mechanism and In Vitro and In Vivo Correlations (IVIVC) were discussed. Kamal Midha discussed the conduct of a bioequivalence study and provided proof of concept for current world wide regulatory standards for $\mathrm{BE}$.

Sabine Kopp provided the World Health Organization (WHO) perspectives on product quality. She outlined WHO's strategy and process for improving the acceptance of generics by health professionals and the public.

Regulations dealing with National Policy leading to professional and public acceptance, WHO guidelines to establish interchangeability (1996) and selection of a comparator product (2002) were discussed.

On both days significant time was devoted to panel discussions with $Q \& A$. Each presentation and $Q$ \& A was translated into Chinese. This was found to be very useful, and key to the success of the workshop. At the end Vinod Shah thanked the organizers for their arrangements and hospitality. Dr. Jin in turn thanked the faculty for participating in this important global outreach activity and indicated that it was very useful to the participants. All participants were provided with an FIP certificate of attendance.

The entire Beijing workshop was repeated in Shanghai, China, April 15-16, 2004. The organization of the Shanghai workshop was primarily done by Dr. Yuming Fang of the Shanghai Institute of Drug Quality Control. In Shanghai, the welcome address was given by Jiye Huang (CCPIE),

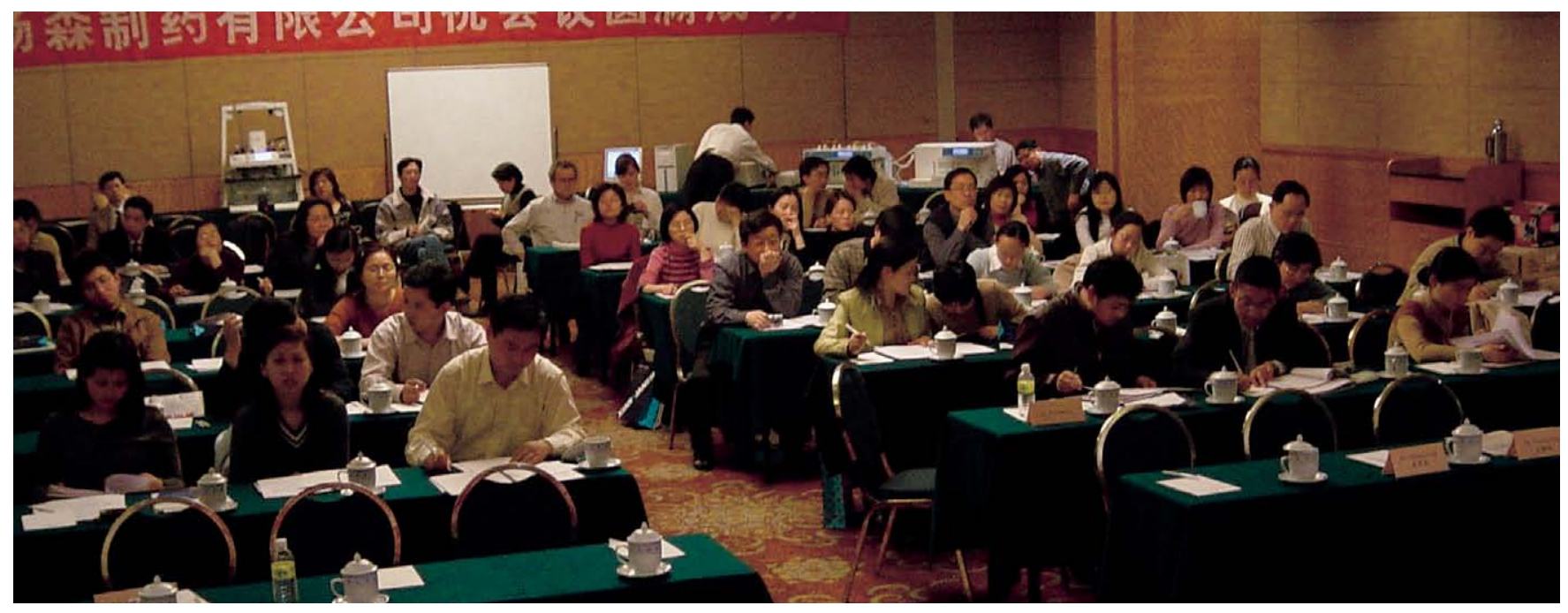

Conference participants in Shanghai 


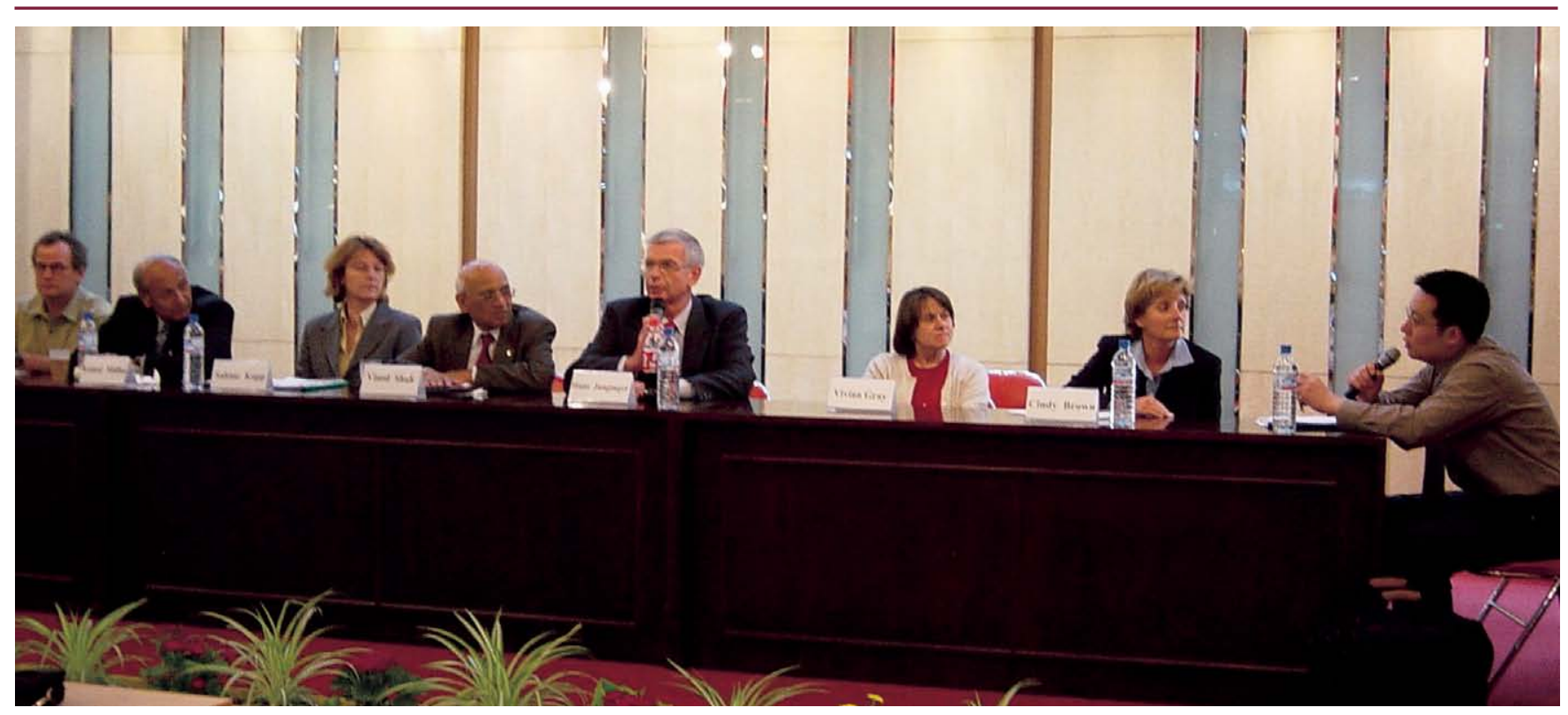

Chengdong Yi (SFDA), Professor Jin, Vinod Shah and Sabine Kopp.

The workshops in Beijing and Shanghai were attended by 130 and 80 scientists respectively. All participants received a diploma of attendance with the FIP logo and signed by the workshop chairs. The workshops were successful and were appreciated by the local organizations. It was a successful outreach global activity of FIP. 